\title{
TRPV2 channel as a possible drug target for the treatment of heart failure
}

\author{
Yuko Iwata $^{1} \cdot$ Shin Ito $^{1} \cdot$ Shigeo Wakabayashi ${ }^{2} \cdot$ Masafumi Kitakaze $^{1}$
}

Received: 29 September 2019 / Revised: 9 November 2019 / Accepted: 13 November 2019 / Published online: 19 December 2019

(c) The Author(s), under exclusive licence to United States and Canadian Academy of Pathology 2019

\begin{abstract}
Heart transplantation is currently the only viable option available for the treatment of severe heart failure conditions such as dilated cardiomyopathy. Hence, novel drugs for treating such conditions need to be developed urgently. Recent studies suggest that $\mathrm{Ca}^{2+}$ overload is involved in the onset and progression of dilated cardiomyopathy, and thus heart failure. The expression and activation of the $\mathrm{Ca}^{2+}$ permeable channel, transient receptor potential vanilloid 2 (TRPV2) channel have been found to play an essential role in sustained intracellular $\mathrm{Ca}^{2+}$ concentration increase, leading to heart failure. However, since there have been no TRPV2-specific inhibitors available until recently, the effect of TRPV2 inhibition on the pathology has not been clearly elucidated. Recent reports show that inhibiting TRPV2 activity effectively improves cardiac function, suppressing myocardial fibrosis and ameliorating the prognosis in animal models of cardiomyopathy with heart failure. In addition to that, inflammation is reported to be involved in the development of heart failure. Here, we review the recent findings on TRPV2 in cardiomyocytes and immune cells involved in the development of heart failure and discuss the current progress of drug development for the treatment of heart failure via targeting TRPV2.
\end{abstract}

\section{Introduction}

When the heart is excessively stressed by intrinsic or extrinsic factors, such as genetic factors, high blood pressure, and ischemia, it reaches a situation wherein it cannot supply blood adequately to meet the body's demand. This situation is termed heart failure (HF), which is a lifethreatening disease that often presents systemic symptoms, such as general fatigue, edema, and dyspnea (https://www. mayoclinic.org/diseases-conditions/heart-failure/symptomscauses/syc-20373142). HF is a chronic disease and is increasing in prevalence, and about 64 million people worldwide suffer from HF; $~ 50 \%$ of patients with HF die within 5 years of diagnosis, which is an outcome similar to that of some malignancies [1]. Dilated cardiomyopathy (DCM) is one of the diseases that lead to HF. While

Yuko Iwata

yukoiwat@ncvc.go.jp

1 Department of Clinical Research and Development, National Cerebral and Cardiovascular Center, Suita, Japan

2 Department of Pharmacology, Osaka Medical Collage, Takatsuki, Osaka, Japan
$30-48 \%$ of DCM cases are inherited, caused by genetic mutations [2], most of the remaining cases are idiopathic (of unknown cause). Among the inherited cases, cytoskeletal protein abnormalities, especially those caused by genetic abnormalities in dystrophin and related proteins, are well known. Some of the genes responsible for these abnormalities are identical to those responsible for muscular dystrophy. Indeed, muscular dystrophy with abnormal skeletal muscle is caused by the same gene abnormality that causes DCM. For example, Duchenne muscular dystrophy develops attributable to dystrophin deficiency, however, a high incidence of combined myocardial dysfunction that leads to DCM. Since ventilators are widely used to control respiratory failure in patients with muscular dystrophy, HF has become an increasingly common cause of death in such patients nowadays. At present, renin angiotensin-converting enzyme inhibitors (ACE-I), angiotensin receptor blockers (ARB), antialdosterone drugs, and $\beta$-blockers are used as the standard treatment for HF, including DCM [3-5]. These drugs are expected to suppress the signals that are activated in cardiac dysfunction and thus alleviate myocardial damage. Patients with HF, who do not respond to conventional drug therapy, require heart transplantation or left ventricular (LV) assist devices because of worsening HF. However, it is challenging to receive a donor heart within 
the appropriate time frame because of the limited number of donors or incompatibility. Therefore, alternative and efficient therapies, apart from conventional therapy, are required in the clinical field before heart replacement is earnestly required $[3,4]$.

\section{$\mathrm{Ca}^{2+}$ abnormality and HF}

The heart possesses the pump function in the body that contracts and relaxes through $\mathrm{Ca}^{2+}$ mediated excitation-contraction coupling. $\mathrm{Ca}^{2+}$ plays a critical role in both physiological and pathophysiological calciumdependent signaling activation. In the process of pathological cardiac remodeling from cardiac hypertrophy to the onset of HF, changes in gene expression following the activation of pathological signals lead to cardiomyocyte dysfunction and cell death. $\mathrm{Ca}^{2+}$-dependent signaling proteins such as calcineurin and calmodulin-dependent kinases (CaMK) are known to play an essential role in this pathological myocardial remodeling process, but the cellular mechanisms for the pathological calcium influx involved in its activation and subsequent increase in intracellular $\mathrm{Ca}^{2+}$ concentration have not been elucidated. The $\mathrm{Ca}^{2+}$ influx from voltage-dependent L-type $\mathrm{Ca}^{2+}$ channel and $\mathrm{Ca}^{2+}$ release from ryanodine receptor $(\mathrm{RyR})$ are essential in the process of physiological excitation-contraction of the myocardium. Moreover, myocardial contraction-relaxation is primarily controlled by the $\mathrm{Ca}^{2+}$ transients [6]. Both L-type $\mathrm{Ca}^{2+}$ channel and RyR coupling are reported to be impaired in failing hearts. Indeed, both increases in $\mathrm{Ca}^{2+}$ leakage and decreases in $\mathrm{Ca}^{2+}$ reuptake in sarcoplasmic reticulum (SR) are suggested to reduce the $\mathrm{Ca}^{2+}$ content in $\mathrm{SR}$ and provoke the intracellular $\mathrm{Ca}^{2+}$ overload, resulting in decreased myocardial contractility and arrhythmia [7]. Recent reports have suggested that CaMKII plays a central role in the pathogenesis of $\mathrm{HF}$ through its role in the molecular mechanisms of HF progression [8], and several studies have been conducted to clarify the role of the $\mathrm{Ca}^{2+}$ influx in the activation of CaMKII.

\section{TRPV2 and cardiomyopathy/HF}

We have studied the pathophysiological cellular mechanisms of cardiomyopathy through the analysis of the abnormalities of $\mathrm{Ca}^{2+}$ mobilization in myogenic cells of DCM animal models with cytoskeletal abnormalities. We found that a sustained increase in intracellular $\mathrm{Ca}^{2+}$ concentration is an essential risk factor for muscle degeneration and that TRPV2 is involved in this process [9]. TRPV2 is a stretch-sensitive channel and its molecular entity has been clarified for the first time in animal cells [9, 10]. It responds with growth factor such as IGF-1 and is activated by translocation from intracellular vesicles to the plasma membrane (Fig. 1). Interestingly, it has been reported that levels of both TRPV2 protein and mRNA increase in HF patients, and this has been expressed in the sarcolemma of cardiomyocytes in human DCM hearts [11]. Conversely, TRPV2 expression has not been observed in the sarcolemma of normal cardiomyocytes and has been detected in the intracellular membrane system or intercalated discs [11]. In several appropriate animal models of human DCM, such as DCM hamsters (J2N-k), knock in mice (TNNT2 $\Delta \mathrm{K} 210$ ) with cardiac troponin T mutation [12], and DCM mice with abnormal sugar chain (4C30) [13], we have found increased expression of TRPV2 in the sarcolemma and increased $\mathrm{Ca}^{2+}$ influx via TRPV2 [11]. Besides, both activation of CaMKII and reactive oxygen species (ROS) production leading to HF are suggested to be induced by the continuous increase in intracellular $\mathrm{Ca}^{2+}$ concentration via TRPV2 [11]. Transgenic (Tg) mice overexpressing TRPV2 in the myocardial sarcolemma have also been shown to develop DCM due to muscle degeneration with $\mathrm{Ca}^{2+}$ overload [9]. In addition to our group, several other groups have reported the involvement of TRPV2 in muscular dystrophy cardiomyopathy. Aguettaz et al. used the cardiac myocytes isolated from older mdx mice (10-12 months old) to evaluate the localization and the pathological function of TRPV2 [14]. This report showed that TRPV2 located in the sarcolemma causes cation influx and subsequent dysregulation in dystrophin-deficient cardiomyocytes and is often enhanced under stretching conditions. On the other hand, Lorin et al. reported the abnormal $\mathrm{Ca}^{2+}$ signals via TRPV2 in mdx cardiomyocytes during hypoosmotic stress [15]. Furthermore, recent studies using rodent models of transverse aortic constriction (TAC)-induced cardiac hypertrophy [16] or $\mathrm{HF}$, myocardial ischemia-reperfusion (MI/R) injury, myocardial infarction (MI) [17, 18], and medicationinduced cardiomyopathy [11] showed that enhanced TRPV2 expression was associated with cardiac dysfunction. Indeed, TRPV2 is reported to play an essential role in HF (Table 1). In the case of rat MI/R injury, TRPV2 is upregulated through the downregulation of miR202-5p [19], indicating that TRPV2 could be a promising target for injury attenuation. To confirm that TRPV2 is the therapeutic target for HF, it is crucial to determine whether the specific inhibition of TRPV2 activity could rescue HF. The inhibitory effects using various models have been investigated, the details of which can be found in Table 1 .

There are two strategies for blocking TRPV2 signaling: blocking its accumulation in the plasma membrane (stimulating internalization) or inhibiting $\mathrm{Ca}^{2+}$ influx across the plasma membrane (Fig. 1). Among the investigation as how to suppress the accumulation of TRPV2 in the plasma membrane, we found that a part of the amino-terminal domain (NT) of TRPV2 protein plays a role in retaining 

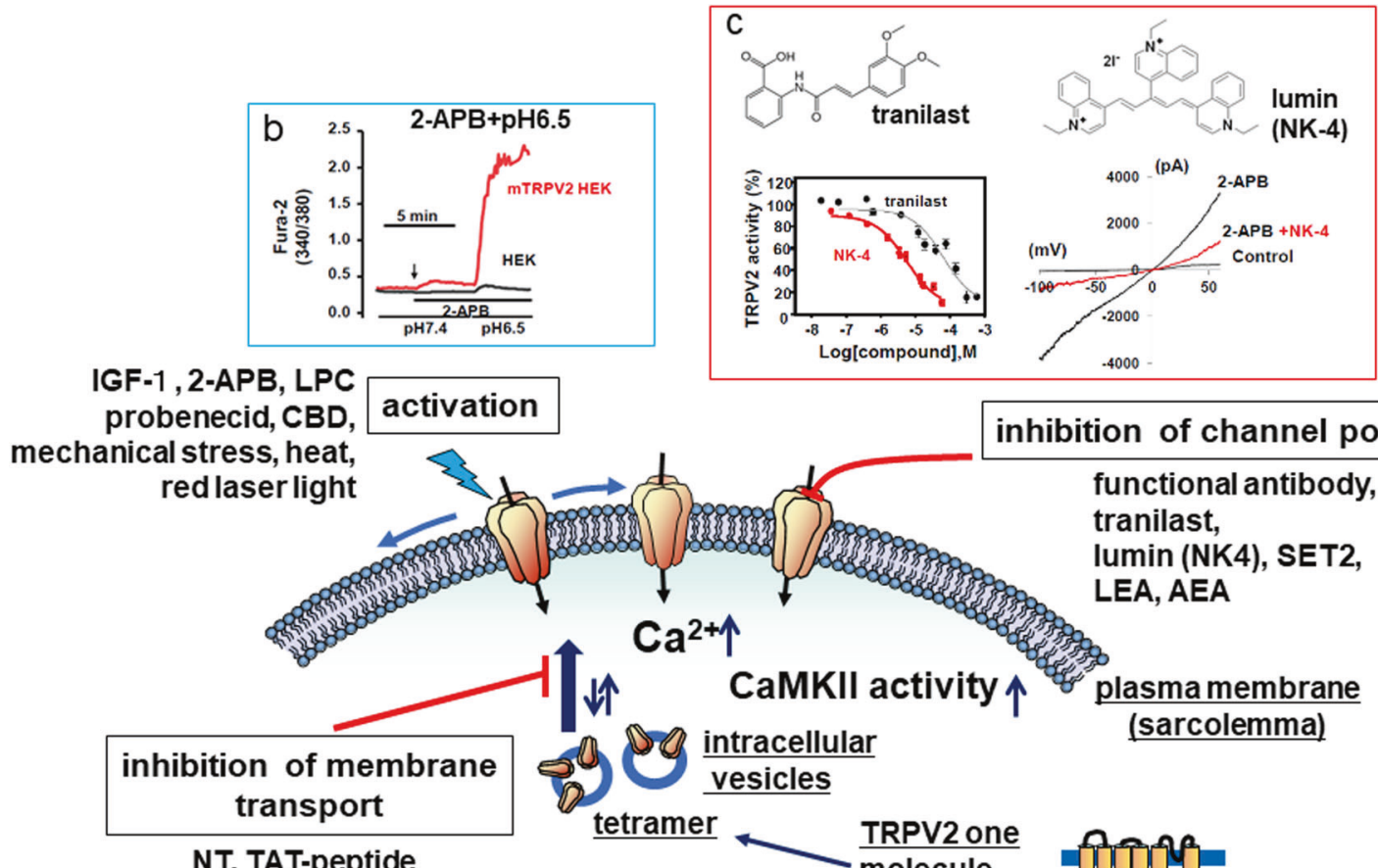

IGF-1 , 2-APB, LPC
probenecid, CBD,

tivation

nical stress, heat,

red laser light

NT, TAT-peptide
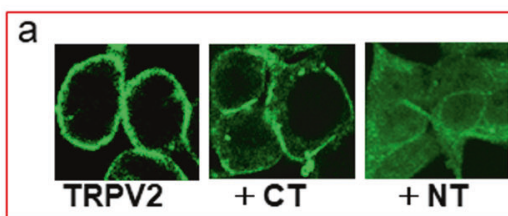

TRPV2 one

inhibition of channel pore

functional antibody,

tranilast,

lumin (NK4), SET2,

LEA, AEA

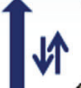

$\mathrm{Ca}^{2+\uparrow}$

CaMKII activity $\uparrow$ plasma membrane

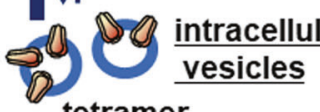

(sarcolemma)

Fig. 1 A schematic drawing for the possible methods to activate and inhibit the $\mathrm{Ca}^{2+}$ influx via TRPV2. TRPV2 forms a tetramer and moves from intracellular vesicles to the plasma membrane (sarcolemma) and exhibits channel activity. IGF-1, 2-aminoethoxydiphenyl borate (2-APB), lysophosphatidylcholine (LPC), probenecid, cannabidiol (CBD), heat $\left(>52^{\circ} \mathrm{C}\right)$, and mechanical stress are known as activators. There are two kinds of methods for inhibiting TRPV2: direct channel inhibition and inhibition of membrane traffic. Direct inhibition can be caused by a functional antibody and TRPV2 inhibitors (tranilast, lumin (NK-4), SET2, LEA, AEA, etc.). Lumin (NK-4); 4,4'-[3-[2-[1-Ethyl-4(1 H)-quinolinylidene]ethylidene]-1-propene-1,3-diyl]bis(1-ethylquinolinium) diiodide, SET2; N-(Furan-2ylmethyl)-3-((4-(N7-methyl-N'-propylamino)-6-(trifluoromethyl)-pyrimidin-2-yl)thio)-propanamide, LEA; linoleoyl ethanolamide, AEA; arachidonoyl ethanolamide. a Immunofluorescence staining of mouse
TRPV2 expressing in HEK293 cells indicated that TRPV2 was found in the plasma membrane. When the amino terminal ( $\mathrm{N}$-terminal) domain (a.a.1-387) of TRPV2 was overexpressed, the intracellular translocation of plasma membrane TRPV2 was promoted. However, overexpression of the carboxy terminal (C-terminal) domain (a.a.633756) of the protein had no such effect. b Mouse TRPV2 (mTRPV2)expressing cells have almost no $\mathrm{Ca}^{2+}$ response at $\mathrm{pH} 7.4$ when 2-APB is used as an activator, but at $\mathrm{pH} 6.5$, they are activated and exhibit an increase in the intracellular $\mathrm{Ca}^{2+}$ concentration several times compared to that found at $\mathrm{pH}$ 7.4. c Structures of tranilast and lumin (NK-4) are shown. Lumin (NK-4) inhibits TRPV2 activity induced by 2-APB using mTRPV2-expressing HEK293 cells at a concentration lower than that of tranilast (left). Moreover, $15 \mu \mathrm{M}$ lumin (NK-4) suppresses $200 \mu \mathrm{M}$ 2-APB-induced current measured by the patch clamp method.

dysfunction and enhances survival in 4C30 DCM mice, TNNT2 $\Delta \mathrm{K} 210$ DCM mice, and J2N-k hamsters by preventing abnormal $\mathrm{Ca}^{2+}$ increase and CaMKII activation. TAC or MI (ischemia-reperfusion of the isolated heart) or adriamycin-induced HF in NT-TRPV2 $\mathrm{Tg}$ mice has also been found to be alleviated by utilizing this therapeutic method [11]. These results suggest that TRPV2 in cardiac sarcolemma plays a role in worsening HF.

Recently, we created an antibody to inhibit TRPV2specific activity from the outside of cells. The administration of this antibody improved not only cardiomyopathic animals [21] but also cardiac hypertrophy and HF in 


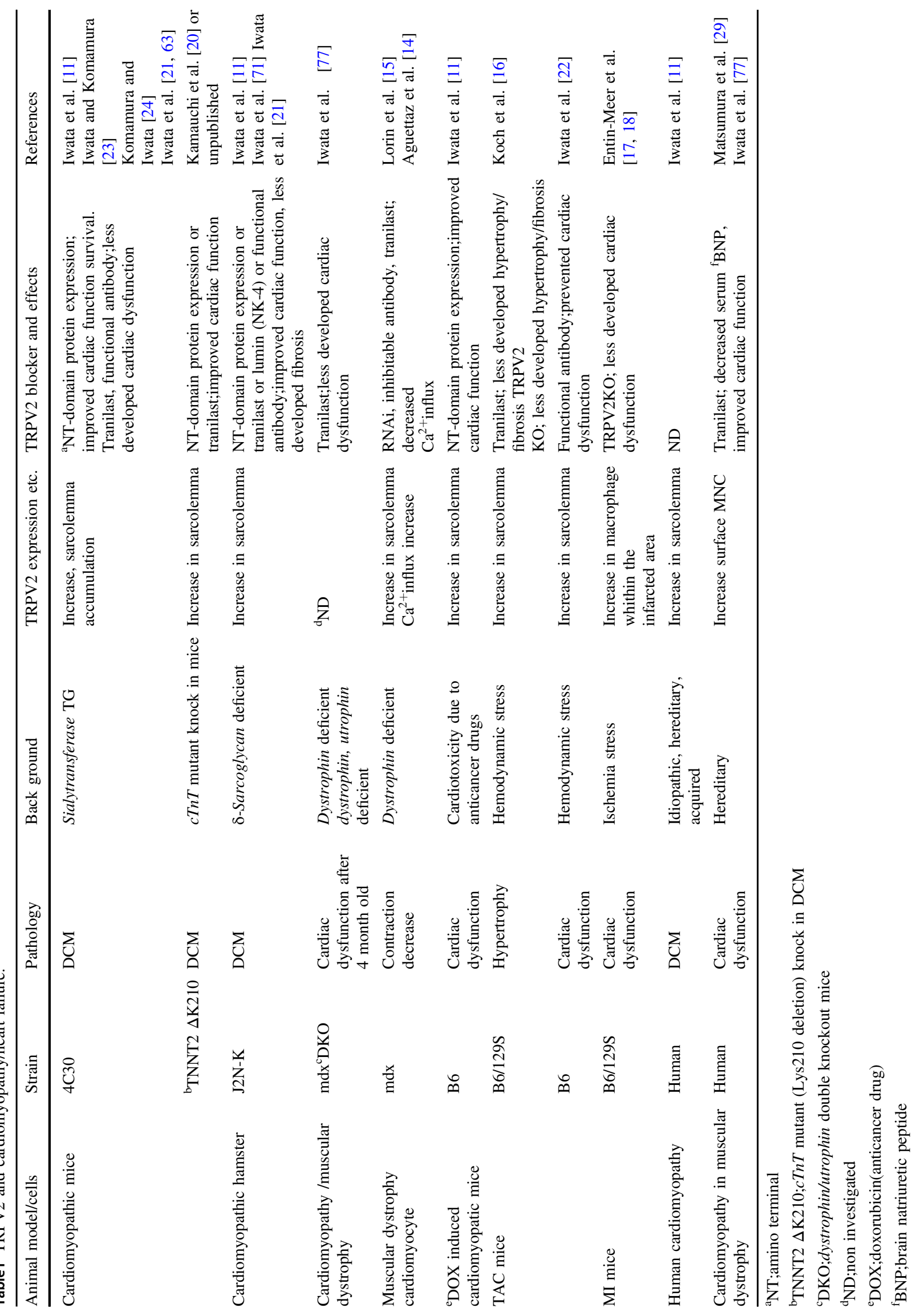


TAC model [22] (Table 1). Using a pore-blocking antibody and/or tranilast as an inhibitor of TRPV2, TRPV2 has been shown to be involved in the abnormal $\mathrm{Ca}^{2+}$ signals in DCM [23, 24], as well as DCM in muscular dystrophy [11, 14, 15].

Besides, the studies using small-interfering RNA and antisense RNA against TRPV2 gene [9] or TRPV2-deficient animals have confirmed the pathological significance of TRPV2. It has been reported that TAC model hearts or MI model hearts in TRPV2 knockout mice (TRPV2KO) mice reduced TRPV2 signals, thus suppressing both cardiac hypertrophy [16] and cardiac dysfunction [18].

\section{HF and Inflammation-an involvement of TRPV2}

Although the importance of inflammation in heart disease, especially MI, and myocarditis was already known, inflammation has been recently identified to also trigger or mediate the development and progression of HF. Patients with $\mathrm{HF}$ have increased serum levels of inflammatory cytokines such as TNF- $\alpha$ and IL- $1 \beta$, as well as in the heart [25]. Inflammatory cytokines can lead to reduced cardiac function and cardiac remodeling in various animal models [26]. Diverse mechanisms such as abnormal calcium handling in cardiomyocytes and the promotion of fibrosis by the activation of fibroblasts are likely to be involved, including TRPV2 [27]. The transcriptomic profile revealed by Hulanicka et al. showed the upregulation of TRPV2 in the peripheral blood nuclear cells in canine HF [28]. We observed that the TRPV2 channel increases in the plasma membrane of peripheral mononucleated cells obtained from patients with cardiomyopathy associated with muscular dystrophy [29]. In the immune system, TRPV2 is involved in both innate and adaptive immune responses [30]. In macrophages, TRPV2 expression is high, and it is the only TRPV channel expressed in these cells [31]. In macrophages, TRPV2 is localized mainly in the endoplasmic reticulum under unstimulated conditions. The stimulation by a chemotactic peptide such as fMetLeuPhe (fMLP), growth factors, or mechanical stress induces the translocation of TRPV2 to the plasma membrane and causes its accumulation in the podosomes, a specific actin-adhesive machinery that regulates adhesion and migration of macrophages [32]. The translocation of TRPV2 is mediated by the receptormediated activation of phosphatidyl-inositol 3-kinase and Rac GTPase, and then the TRPV2 undergoes endocytosis by a mechanism involving a GTPase dynamin [33]. Inhibition of TRPV2 attenuates $\mathrm{Ca}^{2+}$ entry activated by ligands or stress, and blocks the ligand-induced migration of macrophages or stress-induced inflammatory signals [34].

It is well known that macrophages participate in acute and chronic tissue remodeling via phagocytosis and crosstalk with stromal cells. Activation of TRPV2 is indispensable in the earliest steps of macrophage phagocytosis [35]. Further, it was recently reported [36] that macrophages reside in the healthy heart and are recruited in large numbers into acute ischemic tissues; within 2 months after cardiac ischemia, the number of macrophages increase in the remote myocardium due to local macrophage proliferation and/or due to monocyte recruitment [36]. Increased local macrophage proliferation results in part from mechanical stress in failing hearts. About one-third of the recruited peripheral monocytes produced in the bone marrow and spleen contribute to the increase in the number of cardiac macrophages. If monocyte recruitment is diminished, LV remodeling should be attenuated. These data provided casual evidence that such monocyte recruitment contributes to the development and progression of $\mathrm{HF}$ after MI. Recently, it has been shown that the deletion of TRPV2 in mice presents a significantly improved functional recovery following acute MI compared with their WT counterparts and that an intravenous administration of WT macrophages, but not of KO macrophages, reduced survival of post-MI TRPV2-KO mice [18]. These pieces of evidence suggest that TRPV2 is involved in proinflammatory processes as mentioned above and TRPV2 inhibition may attenuate that process, leading to alleviate $\mathrm{HF}$.

The contribution of TRPV2 to the other interesting functionally related phenomena in macrophages, for example, cardiac electrical conduction [37] or M1/M2 polarization and differentiation [38], needs to be explored to gain a better understanding of the role of TRPV2 in heart diseases through immune system. The investigation using mast cell-deficient mice revealed that mast cells in the pathology of $\mathrm{HF}$ are also involved. Systolic pressure overload-induced cardiac dysfunction was prevented through mast cell ablation and similar beneficial effects were observed after treatment with a TRPV2 inhibitor, tranilast [39]. Cell surface expression of TRPV2 with the functional coupling of TRPV2 protein to $\mathrm{Ca}^{2+}$ influx in response to mechanical, heat and red laser light stimulation, and induced release of proinflammatory mediators or degranulation has been reported in mast cells [40-42]. These data suggest that TRPV2 plays a crucial role in mast cell degranulation that is involved in the progression of HF.

\section{HF and other TRPs}

In our previous study, besides TRPV2, TRPC1 protein expression in LV samples was higher in DCM patients than in controls [11]. Recently, it was reported that mRNA levels of TRPC1, 3, 4, and 6 and TRPV2 were increased, and the levels of TRPM2, 3, and 8 were decreased in failing human LV samples [43]. On the other hand, an increase in the mRNA levels of TRPC1, 5, TRPM4 and TRPM7, and a decrease in TRPC4 and TRPV2 levels were found in 
samples taken from end-stage HF patients as compared with myocardial samples from healthy donor hearts [44]. Detailed evidence has confirmed that the activity and expression of several TRP channels are upregulated in pathological hypertrophy and HF as reviewed elsewhere $[41,45,46]$. Briefly, it was reported that TRPC1, 3, and 6 upregulation linked to calcineurin/NFAT signaling in pressure-overload hypertrophy and cardiomyopathy in several rodent models, but it was not mentioned the CaMKII activation led to HF. In a study using DCM mouse model (muscle LIM protein deficiency, MLP-/-), the upregulation of TRPC3, increased cardiomyocyte CaMKII activity, and ROS production were observed, and these signals have been decreased with cardiac functional improvement after treatment with the TRPC3-specific inhibitor [47]. Recently, it has been reported that TRPC1 and TRPC4 together work for a background $\mathrm{Ca}^{2+}$ entry, which determines diastolic and systolic $\mathrm{Ca}^{2+}$ concentrations under neurohumoral stimulation as well as under basal conditions in beating cardiomyocytes, and that TRPC1/C4gene inactivation protects against development of maladaptive cardiac remodeling in mice [48].

TRPV1 has been studied using its KO mice and its agonist/antagonist for a long time, and the data regarding HF have also been discussed [49]. The dual role of TRPV1 has been reported in terms of HF, namely, activation of the TRPV1 channel involved in improving heart function in pathological conditions, including HF [50-53]. TRPV1 channel activation increases the expression of uncoupling protein 2, peroxisome proliferator-activated receptor-delta, and mitochondrial sirtuin 3 to decrease oxidative stress and reduce heart injury [53]. In addition, it has been reported that TRPV1 activation in cardiac sensory neurons and subsequent CGRP release reduces ischemia-reperfusion injury [54]. On the other hand, TRPV1 channel activation has been reported to aggravate HF [55-57]. In these studies, activation of TRPV1 has been reported to enhance the expression of the hypertrophic fibrotic protein to promote cardiac fibrosis.

Recently, TRPV4 was reported to be involved in HF. While mutations of desmin are linked to DCM [58, 59], we recognized that one of the DCM-hiPSC-CM lines that carry a point mutation in the desmin proteins (A285VDES) had shown the abnormal increase in the intracellular $\mathrm{Ca}^{2+}$, which was mostly related to TRPV4 rather than TRPV2 or L-type Ca channels [60]. It was reported that the orally active TRPV4 channel blocker GSK2193874 prevents and resolves HF-induced pulmonary edema, and results in a reduced LV dilation of post-MI upon pretreatment [61]. The importance of TRPV4 in ventricular remodeling has also been confirmed by using TRPV4KO mice [62]. It was reported that in the aged heart, TRPV4 not only enhances cardiomyocyte calcium cycling and contractility, but also contributes to damage following hypoosmotic stress.

\section{TRPV2 as a therapeutic target for HF}

Since safety is most essential when studying drug discovery, we conducted a safety test in which normal mice were administered TRPV2 functional antibody at a sufficient concentration or higher, and no severe side effects were observed [63]. Many laboratories have confirmed that even when TRPV2 is deleted in whole body using TRPV2KO, it does not show a significant decrease in cardiac function or any other severe symptoms [64]. These lines of evidence indicate that inhibition of TRPV2 as a treatment might not affect healthy cells but act specifically on diseased cells. Abnormal $\mathrm{Ca}^{2+}$ transients (significantly higher diastolic $\mathrm{Ca}^{2+}$ levels) were observed under electrical stimulation in isolated cardiomyocytes from rodent DCM hearts, but functional antibodies and tranilast inhibited them by blocking TRPV2 [63]. TRPV2 inhibitor therapies are entirely different from conventional $\mathrm{Ca}^{2+}$ channel inhibitors (voltage-dependent $\mathrm{Ca}^{2+}$ channel blockers), which affect $\mathrm{Ca}^{2+}$ influx via excitation-contraction coupling associated with pulsation. TRPV2 inhibition attenuates the increase of intracellular $\mathrm{Ca}^{2+}$, especially during the diastolic phase, and improves $\mathrm{Ca}^{2+}$ signaling abnormalities, for example, caused by activation of CaMKII, which is thought to lead to worsening of HF. Therefore, the treatment using a TRPV2 inhibitor, combined with standard therapy, could result in more beneficial effects, since the effectors of TRPV2 inhibitors are different from those of standard drugs such as ACE-I, ARB, antialdosterone drugs, and $\beta$-blockers with (in some cases) ionotropic agents [65], $\mathrm{Ca}^{2+}$ sensitivity enhancers [66], or myosin activators [67, 68].

\section{Identification of TRPV2 inhibitors}

Although TRPV2 seems to be an excellent therapeutic target for HF, no specific inhibitors have been identified until recently due to the lack of specific TRPV2-activators and a reproducible TRPV2 activity assay system. To the best of our knowledge, no selective TRPV2 antagonists even from natural sources have been validated so far. The only known ligand for TRPV2 is the natural product cannabidiol (CBD), which is a potent agonist of TRPV2; however, it also targets TRPA1, TRPV1, and other cellular receptors, such as the cannabinoid receptor, serotonin $5-\mathrm{HT}_{1 \mathrm{~A}}$ receptor, GPR55, and PPAR- $\gamma$ [69]. In addition to CBD, probenecid, lysophosphatidylcholine (LPC), 2-aminoethoxy diphenyl borate (2-APB), high temperature $\left(>52^{\circ} \mathrm{C}\right)$, and mechanical stress are also known to activate TRPV2 nonspecifically. Recently, monanchomycalin B, a marine cyclic guanidine alkaloid, has been reported to inhibit 2-APB induced $\mathrm{Ca}^{2+}$ 
response of TRPV2 selectively but it is not TRPV2-specific (IC50 6.02, 2.84, and $3.25 \mu \mathrm{M}$ for TRPV1, TRPV2, and TRPV3, respectively) [70]. We developed an assay system (Fig. 1b) and found several novel compounds, including lumin (NK-4), that inhibited TRPV2 activity with IC50 of $<5 \mu \mathrm{M}$ for the 2-APB-induced $\mathrm{Ca}^{2+}$ response and currents (Fig. 1c) with no effects on TRPV1 and TRPC1 as reported [71]. Recently, using cryo-electron microscopic analysis, putative ligand binding sites of TRPV2 were identified and potential inhibitors targeting these sites were prepared. Among these inhibitors, SET2 was developed as a potent TRPV2-selective antagonist against the 2-APB induced activation $(\mathrm{IC} 50=0.46 \mu \mathrm{M})$ in which the chemical name of SET2 is N-(Furan-2-ylmethyl)-3-((4-(N7-methyl-N'-propylamino)-6-(trifluoromethyl)-pyrimidine-2-yl) thio)-propanamide [72]. As a result of searching for synthetic or endogenous lipids structurally related with capsaicin derivatives, linoleoyl ethanolamide and arachidonoyl ethanolamide (AEA) were discovered as potent TRPV2 antagonists against CBD stimulation (with IC50 of 0.65 and $0.96 \mu \mathrm{M}$, respectively) [73]. AEA is not specific for TRPV2 [74]. Functional antibodies against TRPV2 are more preferred as specific inhibitors. As mentioned above, we succeeded in its production and observed its beneficial effects on DCM animal models [63]. However, the antibody could not recognize the human TRPV2; thus, clinicians require human-specific TRPV2 functional antibody for therapy.

\section{Clinical application for HF therapy targeting TRPV2}

There are several compounds putatively targeting TRPV2 for HF therapy (Fig. 2). Tranilast and other TRPV2

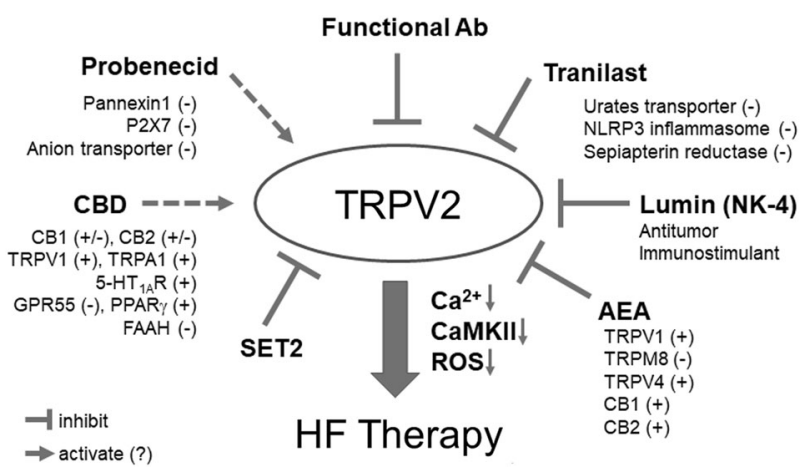

Fig. 2 Compounds that were reported to inhibit or activate TRPV2, possibly targeting heart failure (HF). Various TRPV2 modulators and their other known effectors are shown. $(-)$; inhibit $(+)$ : activate. Inhibition of TRPV2 reduces intracellular $\mathrm{Ca}^{2+}$ abnormality and ameliorates HF. The beneficial effects of tranilast (antiallergy drug), lumin (antioxidant), and functional antibody through TRPV2 inhibition were reported. In addition to TRPV2, several compounds were reported to inhibit $(-)$ or activate $(+)$ other targets, as shown in the figure. At present, tranilast, lumin, $\mathrm{CBD}$, and probenecid can be used in humans but have not yet been used in the treatment of HF. inhibitors including lumin (NK-4), AEA, SET2 could ameliorate the abnormal $\mathrm{Ca}^{2+}$ level increase and $\mathrm{Ca}^{2+}$ signals. Interestingly, both probenecid and CBD, which have been reported as activators of TRPV2 besides their other various actions, have also been reported to show beneficial effects for HF therapy. Their precise mechanisms must be further elucidated.

\section{Tranilast}

The antiallergic drug tranilast has long been reported to be effective for $\mathrm{HF}$ in animals $[39,75,76]$, including canine HF [76]. However, tranilast has not yet been used as HF treatment in clinical settings. In our exploratory study, TRPV2 inhibition therapy was adopted to treat myocardial damage in patients with muscular dystrophy. A clinical evaluation was performed after administering a regular dose of tranilast in two patients with advanced HF in muscular dystrophy. The serum levels of brain natriuretic peptide (BNP), an HF marker, decreased in both patients within 1 month of tranilast treatment [29]. Subsequently, serum BNP level continued to be low even after continuous administration of tranilast for more than 1 year, and an improvement in cardiac function evaluated with fractional shortening (FS) by more than twice as much as before was observed in both patients (one patient exhibited an improvement of FS to 9 from 4\%, the other patient increased to 11 from 6\%) [77]. These effects are presumed to be good despite the low invasiveness. Tranilast has other sound effects, such as inhibitory effects on urate transporters [78], nod-like receptor pyrin domain containing 3 inflammasome [79], and sepiapterin reductase [80] for other diseases.

\section{Probenecid}

Recently, a clinical study reported that probenecid (uricosuric drug), which showed a modulatory activity against TRPV2 [81, 82], was administered to patients with decreased cardiac contractility [83]. Ejection fraction was reported to have slightly improved after 1 week of probenecid administration. In this study, the improvement of cardiac contractility by probenecid was explained by a transient increase in cytosolic $\mathrm{Ca}^{2+}$ concentrations via TRPV2 activation and its direct action on contractile proteins. A cohort study to examine the comparative cardiovascular safety of two drugs used in gout treatment, probenecid and allopurinol, showed that probenecid was associated with a $20 \%$ lower risk of hospitalization for MI or stroke compared with allopurinol in patients with gout [84]. Probenecid is an inhibitor of P2X7 [85] and anion transporter as well as pannexin with IC50 $=150 \mu \mathrm{M}$. Probenecid may exhibit an antiinflammatory effect through its 
inhibition of pannexin 1 [86], thereby reducing IL-1 $\beta$, leading to the observed beneficial effects in the CANTOS trial [87]. Probenecid is also a urate transporter inhibitor [88] and lowers serum uric acid by blocking the reuptake of uric acid in the kidneys. Further studies are needed to clarify the therapeutic target of probenecid for HF.

\section{CBD}

Recently, it has been reported that CBD attenuates cardiac dysfunction and oxidative stress in diabetic cardiomyopathy (https://clinicaltrials.gov/ct2/show/NCT03634189) [89]. A clinical trial using CBD for patients with HF has been initiated (https://www.drugbank.ca/drugs/DB07615). However, CBD had not been thought as a TRPV2 modulator in this study. Recently, CBD has been reported to bind to TRPV2 and activate it [90] like TRPV1, but CBD has also been reported to have diverse pharmacological effects, which are reviewed in depth elsewhere [69]. In brief, CBD shows antagonism to classical cannabinoid 1 and cannabinoid 2 receptors in the low nanomolar ranges, yet has agonist/inverse agonist actions at micromolar concentrations. It was also found that CBD inhibits both AEA hydrolysis through fatty acid amide hydrolase [91], and AEA uptake by transporter [92]. This means that CBD can increase serum levels of AEA, which is a potent endogenous inhibitor of TRPV2 as mentioned above. AEA is reported to be protective against ischemic injury or HF $[93,94]$. In fact, it was shown that CBD inhibited fMLPstimulated neutrophil migration [95] and white blood cell migration in the cerebral blood vessels after lipopolysaccharide treatment [96]. These pathways may involve the TRPV2-inhibitory effects of CBD, suggesting that CBD may act as a TRPV2 inhibitor as well, although it may be indirect.

\section{Conclusion}

Recent research on TRPV2 has made remarkable progress, especially in terms of the discovery of specific inhibitors and endogenous inhibitors. Further progress in this field can be expected with several clinical trials aimed at the treatment of HF with drugs targeting TRPV2. Regarding HF, it is required to understand the disease in relation to not only the heart but also the kidney, brain, blood vessels, and immune system. Similarly, it is necessary to consider the role of TRPV2 in various organs systematically. From this point of view, the development of therapeutic agents for HF targeting TRPV2 should be promoted. We hope interventions targeting TRPV2 will yield new therapies. Particularly, TRPV2 inhibition therapy using a functional TRPV2 antibody is expected as future medication for HF.
Acknowledgements We thank Dr Ryu Nagata, Osaka University for his helpful discussion in this review. This work was funded by the Japan Society for the Promotion of Science Grant-in Aid for Scientific Research (C) [grant number 17k0598] and Grant-in-Aid for Research on Nervous and Mental Disorder of NCNP [grant number 28-6].

\section{Compliance with ethical standards}

Conflict of interest The authors declare that they have no conflict of interest.

Publisher's note Springer Nature remains neutral with regard to jurisdictional claims in published maps and institutional affiliations.

\section{References}

1. Mamas MA, Sperrin M, Watson MC, Coutts A, Wilde K, Burton $\mathrm{C}$, et al. Do patients have worse outcomes in heart failure than in cancer? A primary care-based cohort study with 10-year follow-up in Scotland. Eur J Heart Fail. 2017;19:1095-104.

2. Seidman JG, Seidman C. The genetic basis for cardiomyopathy: from mutation identification to mechanistic paradigms. Cell. 2001;104:557-67.

3. Yancy CW, Jessup M, Bozkurt B, Butler J, Casey DE Jr., Drazner $\mathrm{MH}$, et al. $2013 \mathrm{ACCF} / \mathrm{AHA}$ guideline for the management of heart failure: a report of the American College of Cardiology Foundation/American Heart Association Task Force on Practice Guidelines. J Am Coll Cardiol. 2013;62:e147-239.

4. Ponikowski P, Voors AA, Anker SD, Bueno H, Cleland JG, Coats AJ, et al. 2016 ESC Guidelines for the diagnosis and treatment of acute and chronic heart failure: The Task Force for the diagnosis and treatment of acute and chronic heart failure of the European Society of Cardiology (ESC). Developed with the special contribution of the Heart Failure Association (HFA) of the ESC. Eur J Heart Fail. 2016;18:891-975.

5. Zannad F, Gattis Stough W, Rossignol P, Bauersachs J, McMurray JJ, Swedberg K, et al. Mineralocorticoid receptor antagonists for heart failure with reduced ejection fraction: integrating evidence into clinical practice. Eur Heart J. 2012;33: 2782-95.

6. Eisner DA, Caldwell JL, Kistamas K, Trafford AW. Calcium and excitation-contraction coupling in the heart. Circ Res. 2017;121:181-95.

7. Zima AV, Bovo E, Mazurek SR, Rochira JA, Li W, Terentyev D. $\mathrm{Ca}$ handling during excitation-contraction coupling in heart failure. Pflugers Arch. 2014;466:1129-37.

8. Anderson ME, Brown JH, Bers DM. CaMKII in myocardial hypertrophy and heart failure. J Mol Cell Cardiol. 2011;51: 468-73.

9. Iwata Y, Katanosaka Y, Arai Y, Komamura K, Miyatake K, Shigekawa M. A novel mechanism of myocyte degeneration involving the $\mathrm{Ca}^{2+}$-permeable growth factor-regulated channel. J Cell Biol. 2003;161:957-67.

10. Muraki K, Iwata Y, Katanosaka Y, Ito T, Ohya S, Shigekawa M, et al. TRPV2 is a component of osmotically sensitive cation channels in murine aortic myocytes. Circ Res. 2003;93:829-38.

11. Iwata Y, Ohtake H, Suzuki O, Matsuda J, Komamura K, Wakabayashi S. Blockade of sarcolemmal TRPV2 accumulation inhibits progression of dilated cardiomyopathy. Cardiovasc Res. 2013;99:760-8.

12. Du CK, Morimoto S, Nishii K, Minakami R, Ohta M, Tadano N, et al. Knock-in mouse model of dilated cardiomyopathy caused by troponin mutation. Circ Res. 2007;101:185-94. 
13. Suzuki O, Kanai T, Nishikawa T, Yamamoto Y, Noguchi A, Takimoto K, et al. Adult onset cardiac dilatation in a transgenic mouse line with Galbeta1,3GalNAc alpha2,3-sialyltransferase II (ST3Gal-II) transgenes: a new model for dilated cardiomyopathy. Proc Jpn Acad Ser B Phys Biol Sci. 2011;87:550-62.

14. Aguettaz E, Lopez JJ, Krzesiak A, Lipskaia L, Adnot S, Hajjar RJ, et al. Axial stretch-dependent cation entry in dystrophic cardiomyopathy: Involvement of several TRPs channels. Cell Calcium. 2016;59:145-55.

15. Lorin C, Vogeli I, Niggli E. Dystrophic cardiomyopathy: role of TRPV2 channels in stretch-induced cell damage. Cardiovasc Res. 2015;106:153-62.

16. Koch SE, Mann A, Jones S, Robbins N, Alkhattabi A, Worley $\mathrm{MC}$, et al. Transient receptor potential vanilloid 2 function regulates cardiac hypertrophy via stretch-induced activation. J Hypertens. 2017;35:602-11.

17. Entin-Meer M, Levy R, Goryainov P, Landa N, Barshack I, Avivi $\mathrm{C}$, et al. The transient receptor potential vanilloid 2 cation channel is abundant in macrophages accumulating at the peri-infarct zone and may enhance their migration capacity towards injured cardiomyocytes following myocardial infarction. PLoS ONE. 2014;9: e105055.

18. Entin-Meer M, Cohen L, Hertzberg-Bigelman E, Levy R, BenShoshan J, Keren G. TRPV2 knockout mice demonstrate an improved cardiac performance following myocardial infarction due to attenuated activity of peri-infarct macrophages. PLoS ONE. 2017;12:e0177132.

19. Li Y, Li Q, Zhang O, Guan X, Xue Y, Li S, et al. miR-202-5p protects rat against myocardial ischemia reperfusion injury by downregulating the expression of Trpv2 to attenuate the $\mathrm{Ca}(2+)$ overload in cardiomyocytes. J Cell Biochem. 2019;120:13680-93.

20. Kamauchi S, Iwata Y, Du C-K, Zhan D-Y, Morimoto S, Shirai M, et al. Overexpression of the amino-terminal domain of TRPV2 has the beneficial effect for dilated cardiomyopathy mice with mutation of troponin T. J Pharmacol Sci. 2014;124:194.

21. Iwata Y, Hirayama M, Ito S, Kitakaze M. Treatment with TRPV2 antibody ameliorates the severity of heart failure in dilated cardiomyopathic hamsters. In: 18th World Congress of Basic and Clinical Pharmacology. WCP2018Kyoto. Japan (Kyoto), 2018.

22. Iwata Y, Hirayama M, Ito S, Kitakaze M. Inhibition of TRPV2 prevents the progression of murine heart failure. In: The 92nd Annual Meeting of the Japanese Pharmacological Society. Japan (Osaka), 2019.

23. Iwata Y, Komamura K. Tranilast, Transient receptor potential vanilloid 2 antagonist, ameliorates end-stage heart failure of mice with dilated cardiomyopathy. Circulation. 2014;130:11977.

24. Komamura K, Iwata Y. Tranilast, orally active TRPV2 antagonist, ameliorates end-stage heart failure in mice with dilated cardiomyopathy. J Cardiac Fail. 2014;20:S198-9.

25. Hedayat M, Mahmoudi MJ, Rose NR, Rezaei N. Proinflammatory cytokines in heart failure: double-edged swords. Heart Fail Rev. 2010;15:543-62.

26. Gullestad L, Ueland T, Vinge LE, Finsen A, Yndestad A, Aukrust P. Inflammatory cytokines in heart failure: mediators and markers. Cardiology. 2012;122:23-35.

27. Santoni G, Morelli MB, Amantini C, Santoni M, Nabissi M, Marinelli $\mathrm{O}$, et al. "Immuno-transient receptor potential ion channels": the role in monocyte- and macrophage-mediated inflammatory responses. Front Immunol. 2018;9:1273.

28. Hulanicka M, Garncarz M, Parzeniecka-Jaworska M, Jank M. The transcriptomic profile of peripheral blood nuclear cells in dogs with heart failure. BMC Genomics. 2014;15:509.

29. Matsumura T, Matsui M, Iwata Y, Asakura M, Saito T, Fujimura $\mathrm{H}$, et al. A pilot study of tranilast for cardiomyopathy of muscular dystrophy. Intern Med. 2018;57:311-8.
30. Santoni G, Farfariello V, Liberati S, Morelli MB, Nabissi M, Santoni M, et al. The role of transient receptor potential vanilloid type- 2 ion channels in innate and adaptive immune responses. Front Immunol. 2013;4:34.

31. Yamashiro K, Sasano T, Tojo K, Namekata I, Kurokawa J, Sawada N, et al. Role of transient receptor potential vanilloid 2 in LPS-induced cytokine production in macrophages. Biochem Biophys Res Commun. 2010;398:284-9.

32. Nagasawa M, Nakagawa Y, Tanaka S, Kojima I. Chemotactic peptide fMetLeuPhe induces translocation of the TRPV2 channel in macrophages. J Cell Physiol. 2007;210:692-702.

33. Nagasawa M, Kojima I. Translocation of calcium-permeable TRPV2 channel to the podosome: Its role in the regulation of podosome assembly. Cell Calcium. 2012;51:186-93.

34. Nagasawa M, Kojima I. Translocation of TRPV2 channel induced by focal administration of mechanical stress. Physiol Rep. 2015;3: e12296.

35. Link TM, Park U, Vonakis BM, Raben DM, Soloski MJ, Caterina MJ. TRPV2 has a pivotal role in macrophage particle binding and phagocytosis. Nat Immunol. 2010;11:232-9.

36. Sager HB, Hulsmans M, Lavine KJ, Moreira MB, Heidt T, Courties G, et al. Proliferation and recruitment contribute to myocardial macrophage expansion in chronic heart failure. Circ Res. 2016;119:853-64.

37. Hulsmans M, Clauss S, Xiao L, Aguirre AD, King KR, Hanley A, et al. Macrophages facilitate electrical conduction in the heart. Cell. 2017;169:510-22 e20.

38. Ma Y, Mouton AJ, Lindsey ML. Cardiac macrophage biology in the steady-state heart, the aging heart, and following myocardial infarction. Transl Res. 2018;191:15-28.

39. Hara M, Ono K, Hwang MW, Iwasaki A, Okada M, Nakatani K, et al. Evidence for a role of mast cells in the evolution to congestive heart failure. J Exp Med. 2002;195:375-81.

40. Stokes AJ, Shimoda LM, Koblan-Huberson M, Adra CN, Turner H. A TRPV2-PKA signaling module for transduction of physical stimuli in mast cells. J Exp Med. 2004;200:137-47.

41. Freichel M, Almering J, Tsvilovskyy V. The role of TRP proteins in mast cells. Front Immunol. 2012;3:150.

42. Zhang D, Spielmann A, Wang L, Ding G, Huang F, Gu Q, et al. Mast-cell degranulation induced by physical stimuli involves the activation of transient-receptor-potential channel TRPV2. Physiol Res. 2012;61:113-24.

43. Morine KJ, Paruchuri V, Qiao X, Aronovitz M, Huggins GS, DeNofrio D, et al. Endoglin selectively modulates transient receptor potential channel expression in left and right heart failure. Cardiovasc Pathol. 2016;25:478-82.

44. Dragun M, Gazova A, Kyselovic J, Hulman M, Matus M. TRP channels expression profile in human end-stage heart failure. Medicina. 2019;55:e380.

45. Yue Z, Xie J, Yu AS, Stock J, Du J, Yue L. Role of TRP channels in the cardiovascular system. Am J Physiol Heart Circ Physiol. 2015;308:H157-82.

46. Hof T, Chaigne S, Recalde A, Salle L, Brette F, Guinamard R. Transient receptor potential channels in cardiac health and disease. Nat Rev Cardiol. 2019;16:344-60.

47. Kitajima N, Watanabe K, Morimoto S, Sato Y, Kiyonaka S, Hoshijima M, et al. TRPC3-mediated $\mathrm{Ca}^{2+}$ influx contributes to Rac1-mediated production of reactive oxygen species in MLPdeficient mouse hearts. Biochem Biophys Res Commun. 2011;409:108-13.

48. Camacho Londono JE, Tian Q, Hammer K, Schroder L, Camacho Londono J, Reil JC, et al. A background $\mathrm{Ca}^{2+}$ entry pathway mediated by TRPC1/TRPC4 is critical for development of pathological cardiac remodelling. Eur Heart J. 2015;36:2257-66.

49. Randhawa PK, Jaggi AS. TRPV1 channels in cardiovascular system: A double edged sword? Int J Cardiol. 2017;228:103-13. 
50. Gao F, Liang Y, Wang X, Lu Z, Li L, Zhu S, et al. TRPV1 activation attenuates high-salt diet-induced cardiac hypertrophy and fibrosis through PPAR-delta upregulation. PPAR Res. 2014;2014:491963.

51. Wang Q, Ma S, Li D, Zhang Y, Tang B, Qiu C, et al. Dietary capsaicin ameliorates pressure overload-induced cardiac hypertrophy and fibrosis through the transient receptor potential vanilloid type 1. Am J Hypertens. 2014;27:1521-9.

52. Wang HJ, Wang W, Cornish KG, Rozanski GJ, Zucker IH. Cardiac sympathetic afferent denervation attenuates cardiac remodeling and improves cardiovascular dysfunction in rats with heart failure. Hypertension. 2014;64:745-55.

53. Lang H, Li Q, Yu H, Li P, Lu Z, Xiong S, et al. Activation of TRPV1 attenuates high salt-induced cardiac hypertrophy through improvement of mitochondrial function. $\mathrm{Br} \mathrm{J}$ Pharmacol. 2015;172:5548-58.

54. Randhawa PK, Jaggi AS. A review on potential involvement of TRPV1 channels in ischemia-reperfusion injury. J Cardiovasc Pharmacol Ther. 2018;23:38-45.

55. Buckley CL, Stokes AJ. Mice lacking functional TRPV1 are protected from pressure overload cardiac hypertrophy. Channels. 2011;5:367-74.

56. Horton JS, Buckley CL, Stokes AJ. Successful TRPV1 antagonist treatment for cardiac hypertrophy and heart failure in mice. Channels. 2013;7:17-22.

57. Lu S, Xu D. Cold stress accentuates pressure overload-induced cardiac hypertrophy and contractile dysfunction: role of TRPV1/ AMPK-mediated autophagy. Biochem Biophys Res Commun. 2013;442:8-15.

58. Li D, Tapscoft T, Gonzalez O, Burch PE, Quinones MA, Zoghbi WA, et al. Desmin mutation responsible for idiopathic dilated cardiomyopathy. Circulation. 1999;100:461-4.

59. Wahbi K, Behin A, Charron P, Dunand M, Richard P, Meune C, et al. High cardiovascular morbidity and mortality in myofibrillar myopathies due to DES gene mutations: a 10-year longitudinal study. Neuromuscul Disord. 2012;22:211-8.

60. Lu J, Lee YK, Ran X, Lai WH, Li RA, Keung W, et al. An abnormal TRPV4-related cytosolic $\mathrm{Ca}(2+)$ rise in response to uniaxial stretch in induced pluripotent stem cells-derived cardiomyocytes from dilated cardiomyopathy patients. Biochim Biophys Acta Mol Basis Dis. 2017;1863:2964-72.

61. Thorneloe KS, Cheung M, Bao W, Alsaid H, Lenhard S, Jian MY, et al. An orally active TRPV4 channel blocker prevents and resolves pulmonary edema induced by heart failure. Sci Transl Med. 2012;4:159ra48.

62. Jones JL, Peana D, Veteto AB, Lambert MD, Nourian Z, Karasseva NG, et al. TRPV4 increases cardiomyocyte calcium cycling and contractility yet contributes to damage in the aged heart following hypoosmotic stress. Cardiovasc Res. 2019;115: 46-56.

63. Iwata Y, Wakabayashi S, Ito S, Kitakaze M. Production of TRPV2targeting functional antibody ameliorating dilated cardiomyopathy and muscular dystrophy in animal models. Under review.

64. Jones S, Mann A, Worley MC, Fulford L, Hall D, Karani R, et al. The role of transient receptor potential vanilloid 2 channel in cardiac aging. Aging Clin Exp Res. 2017;29:863-73.

65. Goldhaber JI, Hamilton MA. Role of inotropic agents in the treatment of heart failure. Circulation. 2010;121:1655-60.

66. Perrone SV, Kaplinsky EJ. Calcium sensitizer agents: a new class of inotropic agents in the treatment of decompensated heart failure. Int J Cardiol. 2005;103:248-55.

67. Malik FI, Hartman JJ, Elias KA, Morgan BP, Rodriguez H, Brejc $\mathrm{K}$, et al. Cardiac myosin activation: a potential therapeutic approach for systolic heart failure. Science. 2011;331:1439-43.

68. Teerlink JR, Felker GM, McMurray JJ, Solomon SD, Adams KF Jr., Cleland JG, et al. Chronic oral study of myosin activation to increase contractility in heart failure (COSMIC-HF): a phase 2, pharmacokinetic, randomised, placebo-controlled trial. Lancet. 2016;388:2895-903.

69. Stanley CP, Hind WH, O'Sullivan SE. Is the cardiovascular system a therapeutic target for cannabidiol? Br J Clin Pharmacol. 2012;75:313-22.

70. Korolkova Y, Makarieva T, Tabakmakher K, Shubina L, Kudryashova E, Andreev Y, et al. Marine cyclic guanidine alkaloids monanchomycalin B and urupocidin A act as inhibitors of TRPV1, TRPV2 and TRPV3, but not TRPA1 receptors. Mar Drugs. 2017;15:87.

71. Iwata Y, Katayama Y, Okuno Y, Wakabayashi S. Novel inhibitor candidates of TRPV2 prevent damage of dystrophic myocytes and ameliorate against dilated cardiomyopathy in a hamster model. Oncotarget. 2018;9:14042-57.

72. Chai H, Cheng X, Zhou B, Zhao L, Lin X, Huang D, et al. Structure-based discovery of a subtype-selective inhibitor targeting a transient receptor potential vanilloid channel. J Med Chem. 2019;62:1373-84.

73. Schiano Moriello A, Lopez Chinarro S, Novo Fernandez O, Eras J, Amodeo P, Canela-Garayoa R, et al. Elongation of the hydrophobic chain as a molecular switch: discovery of capsaicin derivatives and endogenous lipids as potent transient receptor potential vanilloid channel 2 antagonists. J Med Chem. 2018;61: 8255-81.

74. Muller C, Morales P, Reggio PH. Cannabinoid ligands targeting TRP channels. Front Mol Neurosci. 2019;11:487.

75. Darakhshan S, Pour AB. Tranilast: a review of its therapeutic applications. Pharmacol Res. 2015;91:15-28.

76. Nakatani Y, Nishida K, Sakabe M, Kataoka N, Sakamoto T, Yamaguchi $\mathrm{Y}$, et al. Tranilast prevents atrial remodeling and development of atrial fibrillation in a canine model of atrial tachycardia and left ventricular dysfunction. J Am Coll Cardiol. 2013;61:582-8.

77. Iwata Y, Matsumura T. Blockade of TRPV2 is a novel therapy for cardiomyopathy in muscular dystrophy. Int J Mol Sci. 2019;20: 3844.

78. Mandal AK, Mercado A, Foster A, Zandi-Nejad K, Mount DB. Uricosuric targets of tranilast. Pharmacol Res Perspect. 2017;5: e00291.

79. Huang Y, Jiang H, Chen Y, Wang X, Yang Y, Tao J, et al. Tranilast directly targets NLRP3 to treat inflammasome-driven diseases. EMBO Mol Med. 2018;10:e8689.

80. Moore BJR, Islam, B., Ward, S., Jackson,O., Armitage, R., Blackburn J, et al. Repurposing of tranilast for potential neuropathic pain treatment by inhibition of sepiapterin reductase in the BH4 pathway. ACS Omega. 2019;4:11960-72.

81. Koch SE, Gao X, Haar L, Jiang M, Lasko VM, Robbins N, et al. Probenecid: novel use as a non-injurious positive inotrope acting via cardiac TRPV2 stimulation. J Mol Cell Cardiol. 2012;53:134-44.

82. Mihara H, Suzuki N, Yamawaki H, Tominaga M, Sugiyama T. TRPV2 ion channels expressed in inhibitory motor neurons of gastric myenteric plexus contribute to gastric adaptive relaxation and gastric emptying in mice. Am J Physiol Gastrointest Liver Physiol. 2013;304:G235-40.

83. Robbins N, Gilbert M, Kumar M, McNamara JW, Daly P, Koch SE, et al. Probenecid improves cardiac function in patients with heart failure with reduced ejection fraction in vivo and cardiomyocyte calcium sensitivity in vitro. J Am Heart Assoc. 2018;7: e007148.

84. Kim SC, Neogi T, Kang EH, Liu J, Desai RJ, Zhang M, et al. Cardiovascular risks of probenecid versus allopurinol in older patients with gout. J Am Coll Cardiol. 2018;71:994-1004.

85. Bhaskaracharya A, Dao-Ung P, Jalilian I, Spildrejorde M, Skarratt KK, Fuller SJ, et al. Probenecid blocks human P2X7 receptor- 
induced dye uptake via a pannexin-1 independent mechanism. PLoS ONE. 2014;9:e93058.

86. Silverman W, Locovei S, Dahl G. Probenecid, a gout remedy, inhibits pannexin 1 channels. Am J Physiol Cell Physiol. 2008;295:C761-7.

87. Ridker PM, Thuren T, Zalewski A, Libby P. Interleukin-1beta inhibition and the prevention of recurrent cardiovascular events: rationale and design of the Canakinumab Anti-inflammatory Thrombosis Outcomes Study (CANTOS). Am Heart J. 2011;162: 597-605.

88. Tan PK, Ostertag TM, Miner JN. Mechanism of high affinity inhibition of the human urate transporter URAT1. Sci Rep. 2016;6:34995.

89. Rajesh M, Mukhopadhyay P, Batkai S, Patel V, Saito K, Matsumoto $\mathrm{S}$, et al. Cannabidiol attenuates cardiac dysfunction, oxidative stress, fibrosis, and inflammatory and cell death signaling pathways in diabetic cardiomyopathy. J Am Coll Cardiol. 2010; 56:2115-25.

90. Pumroy RA, Samanta A, Liu Y, Hughes TET, Zhao S, Yudin Y, et al. Molecular mechanism of TRPV2 channel modulation by cannabidiol. 2019. https://doi.org/10.1101/521880.
91. Watanabe K, Kayano Y, Matsunaga T, Yamamoto I, Yoshimura $\mathrm{H}$. Inhibition of anandamide amidase activity in mouse brain microsomes by cannabinoids. Biol Pharm Bull. 1996;19:1109-11.

92. Rakhshan F, Day TA, Blakely RD, Barker EL. Carrier-mediated uptake of the endogenous cannabinoid anandamide in RBL-2H3 cells. J Pharmacol Exp Ther. 2000;292:960-7.

93. Nucci C, Gasperi V, Tartaglione R, Cerulli A, Terrinoni A, Bari $\mathrm{M}$, et al. Involvement of the endocannabinoid system in retinal damage after high intraocular pressure-induced ischemia in rats. Investig Ophthalmol Vis Sci. 2007;48:2997-3004.

94. Hydock DS, Lien CY, Hayward R. Anandamide preserves cardiac function and geometry in an acute doxorubicin cardiotoxicity rat model. J Cardiovasc Pharmacol Ther. 2009;14:59-67.

95. Rajesh M, Mukhopadhyay P, Batkai S, Hasko G, Liaudet L, Drel VR, et al. Cannabidiol attenuates high glucose-induced endothelial cell inflammatory response and barrier disruption. Am J Physiol Heart Circ Physiol. 2007;293:H610-9.

96. Ruiz-Valdepenas L, Martinez-Orgado JA, Benito C, Millan A, Tolon RM, Romero J. Cannabidiol reduces lipopolysaccharideinduced vascular changes and inflammation in the mouse brain: an intravital microscopy study. J Neuroinflamm. 2011;8:5. 